\title{
Optimization of the bay resources in substations based on hierarchical model
}

\author{
Wen-Fei Liu ${ }^{\dagger}$ \\ North China Electric Power University Bejing, China \\ E-mail: liuwenfeitgh@163.com \\ Jun $\mathrm{He}$ \\ State Grid Sichuan Electric Power Company \\ Chengdu Power Supply Company Chengdu, China \\ Cheng-Gang Shi \\ State Grid Chongqing Electric Power Company \\ Chongqing,China \\ Hai-Tao Pan \\ State Grid Sichuan Electric Power Company \\ Chengdu Power Supply Company Chengdu,China \\ Tao Chen \\ State Grid Chongqing Electric Power Company \\ Chongqing,China

$$
\text { Chong Pan }
$$ \\ State Grid Sichuan Electric Power Company \\ Chengdu Power Supply Company Chengdu, China \\ Yi Jiang \\ Tsinghua University Beijing,China \\ Yun-Ting Song \\ China Electric Power Research Institute Beijing,China \\ Yin-Shun Wang \\ North China Electric Power University Beijing, China
}


The increasing access requirements of distribution network in urban centers and the limited bay resources in power grid have formed a great contradiction. Accordingly, the bay resource optimization method based on hierarchical model is proposed in this paper. Outer planning selects attributive substations for loads to balance the substation capacity utilization, inner planning determines the association between load and bays in order to achieve the largest number of idle bays, the final bay resource allocation scheme can achieve maximum bay resource utilization. The practical example simulation results show that this method has good practicability, and can provide reference for the planning and construction of urban distribution network.

Keywords: Urban distribution network; Outlet bay; Hierarchical model; Bay resource optimization.

\section{Introduction}

This template, modified in MS Word 2003 and saved as "Word 97-2003 \& 6.0/95 - RTF" for the PC, provides authors with most of the formatting specifications needed for preparing electronic versions of their papers. All standard paper components have been specified for three reasons: (1) ease of use when formatting individual papers, (2) automatic compliance to electronic requirements that facilitate the concurrent or later production of electronic products, and (3) conformity of style throughout a conference proceedings. Margins, column widths, line spacing, and type styles are built-in; examples of the type styles are provided throughout this document and are identified in italic type, within parentheses, following the example. Some components, such as multileveled equations, graphics, and tables are not prescribed, although the various table text styles are provided. The formatter will need to create these components, incorporating the applicable criteria that follow.

With the rapid development of national economy, urban construction process increasingly is more and more fast, users demand increasingly urgent for power. But there exist serious contradiction between the growing demand for access for distribution network in city center and finite resources of grid spacing[1-3].The case of shortage for grid spacing resources has already seriously affected the user's power supply reliability [4-7]and economic development of the city. How to plan rationaly and scientificly for qualifying bay of existing substations has become the emphasis.

Currently, substation bay resources optimization has been discussed by some scholars. Reference [8] analyzed the using situation of urban distribution bay and listed optimization measures; Against to shortage of urban distribution bay, reference [9] compared and analyzed the advantages and disadvantages of common solutions, proposed the related measures for improving the utilization of bay. But measures mentioned above are more from the perspective of wiring or equipment while neglecting optimization the layout between load and the substation bay. 
Combining engineering practice and considering the number of substation free space and used space load proportion, hierarchical model algorithm is proposed to optimize the layout among substations and further improve the utilization of bay resources for substation with constraints of power supply capacity of substation bay and line power supply radius.

\section{Objective Function}

Predigestion relation among substation, load and wires bay is illustrated by fig. 1 and fig.2:

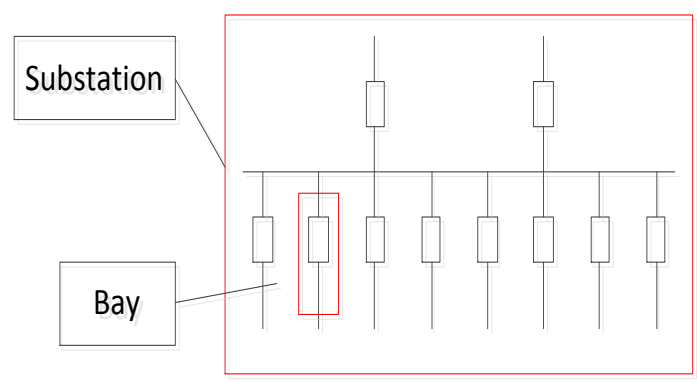

Fig.1 Simplified diagram of the substation and the bay

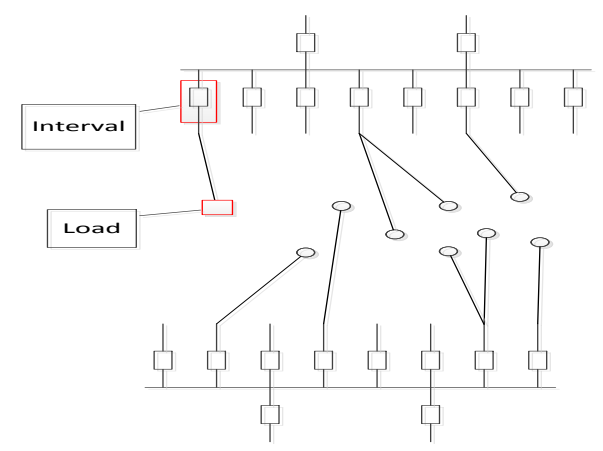

Fig .2 simplified diagram of the load and the spacing

Defining home range matrix between load and substation: CM, the dimension of the matrix is $\left(n_{\text {subl }}+{ }^{\prime} " n_{\text {subm }}\right) \times n_{\text {load }}$, the load is in corresponding bay of power supply radius while element is 1 , the load is not in corresponding bay of power supply radius while element is 0 .where $n_{\text {load }}$ represents the numbers of power supply load in region, $\mathrm{n}_{\text {sub } 1},{ }^{\prime}, n_{\text {subm }}$ represent the numbers of existing bay for every substation.

Defining incidence decision matrix between load and substation: DM. the dimension of the matrix is $\left(n_{\text {subl }}+{ }^{\prime} \cdot \mathrm{n}_{\text {subm }}\right) \times n_{\text {load }}$, power is supplied by corresponding bay when element is 1 ,load and bay has no connection when element 
is 0 . The sum of each column of the matrix must be 1 , which represents there is one and only one bay supplying power for any load.

Decision variables are the elements of matrix DM:

$$
d m_{i j}, d m_{i j} \in\{0,1\}
$$

Where $i=1 \ldots n, j=1$ ' $n_{\text {load }}, n=n_{\text {sub } 2}+n_{\text {sub } 2}+\cdots+n_{\text {subm }}$.

The objective function is maximum of the number for unused bay, that number of rows which elements are not 0 is least:

$$
\min \left(\sum_{i=1}^{n} \max \left\{d m_{i 1}, d m_{i 2}, \ldots, d m_{i n_{\text {load }}}\right\}\right)
$$

Equation restrictions include :1) There is one and only one bay supplying power for one load;2)load fulfill to bay supplying range.

$$
\left\{\begin{array}{l}
\sum_{i=1}^{n} d m_{i j}=1, \quad j=1 \cdots n_{\text {load }} \\
\sum_{i=1}^{n} c m_{i j} d m_{i j}=1, \quad j=1 \cdots n_{\text {load }}
\end{array}\right.
$$

Inequality restrictions include:1) Load carried by each bay does not exceed its capacity;2) each substation load carried does not exceed its capacity.

$$
\left\{\begin{array}{l}
\sum_{j=1}^{n_{\text {load }}} P L_{j} d m_{i j} \leq P_{\text {bayi }}, \quad i=1 \cdots n \\
\sum_{i=1}^{n_{\text {sub } 1}} \sum_{j=1}^{n_{\text {load }}} P L_{j} d m_{i j} \leq P_{\text {sub } 1} \\
\sum_{i=n_{\text {sub } 1}+1}^{n_{\text {sub } 1}+n_{\text {sub } 2}} \sum_{j=1}^{n_{\text {load }}} P L_{j} d m_{i j} \leq P_{\text {sub } 2} \\
\ldots \sum_{i=n_{\text {sub } 1}+\ldots+n_{\text {sub }(m-1)}+1}^{n_{\text {sub } 1}+n_{\text {sub } 2}+. . n_{\text {subm }}} \sum_{j=1}^{n_{\text {load }}} P L_{j} d m_{i j} \leq P_{\text {subm }}
\end{array}\right.
$$

Where PL represents capacity needs for every load; $P_{b a y}$ represents capacity of each bay; $P_{\text {sub }}$ represent total capacity of each substation. 
As the objective function turn into the general form, new integer variables are introduced which number is $\mathrm{n}$ and elements are 0 or $1: \operatorname{dmax}_{1}, \operatorname{dmax}_{2}{ }^{\prime \prime} . \operatorname{dmax}_{n}$. Constraints are introduced at the same time: $d m_{i j} \leq d a m x_{i}, i=1 \ldots n, j=1$ ' $n_{\text {load }}$.

Now, the objective function converts to:

$$
\min \left(\sum_{i=1}^{n} d \max _{i}\right)
$$

Solving the problem is a binary integer linear programming (ILP)[9-12], which includes constraints of:

1) $n \times\left(n_{\text {load }}+1\right)$ integer variables as $\{0,1\}$;

2) $n \times\left(n_{\text {load }}+1\right)+m$ inequality constraints;

3) $2 \times n_{\text {load }}$ equality constraints;

\section{Hierarchical Model}

To reduce the scale of the system, the issue is divided into two layers:(1) load clusters are divided depending on the range of power supply for substation. if load belongs to power supply range of multiple substation, there are needs to optimize substation with the target function of substation capacity balanced utilization to obtain cluster Load of corresponding substation, results is illustrated by Fig.3.(2) For individual substation, based on the capacity of the bay, the objective function is up to the amount of free space to make a strategic decision of connection relation between load and substation and is illustrated by Fig.4.

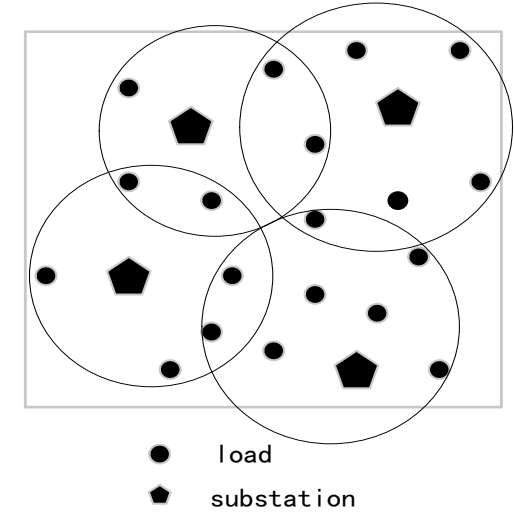

Fig.3 Outer planning diagram 


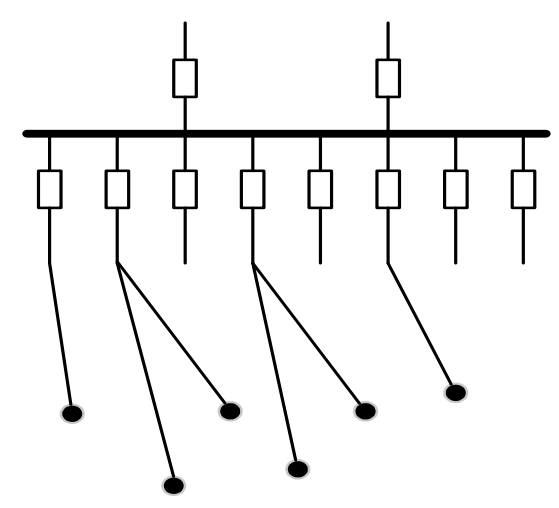

Fig.4 Inner sketch plan

\subsection{Outer planning}

Substation power supply range matrix SM are obtained from CM matrix. The dimension of the matrix is $m \times n_{\text {load }}$. When $s m_{i j}=1$,element represents that load $P L_{j}$ is within the supply range of substation $P_{s u b i}$; when $s m_{i j}=0$,element represents that load $P L_{j}$ is not within the supply range of substation $P_{s u b i}$. Correspondingly, define correlation matrix of load and substation as DSM .

The decision variables is elements of matrix DSM:

$$
d s m_{i j}, d s m_{i j} \in\{0,1\}
$$

Where $i=1 \ldots n, j=1$ ' $n_{\text {load }}$.

The objective function is as follows:

$$
\min \left[\sum_{i=1}^{m}\left(\frac{\sum_{j=1}^{n_{\text {load }}} P L_{j} d s m_{i j}}{P_{\text {subi }}}-\frac{\sum_{j=1}^{n_{\text {load }}} P L_{j}{ }^{2}}{\sum_{i=1}^{m} P_{\text {subi }}}\right)\right]
$$

Equality constraints are:

$$
\left\{\begin{array}{l}
\sum_{i=1}^{m} d s m_{i j}=1, \quad j=1 \cdots n_{\text {load }} \\
\sum_{i=1}^{m} s m_{i j} d s m_{i j}=1, \quad j=1 \cdots n_{\text {load }}
\end{array}\right.
$$

Inequality constraints are: 


$$
\sum_{j=1}^{n_{\text {load }}} P L_{j} d s m_{i j} \leq P_{\text {subi }}, \quad i=1, \cdots, m
$$

In summary, essence of outer optimization model is integer quadratic programming(IQP) problem, which includes constraints of :

1) $m \times n_{\text {load }}$ integer variable as $\{0,1\}$;

2) $m$ inequality constraints;

$3) 2 \times n_{\text {load }}$ equality constraints;

\subsection{Inner planning}

Correlation matrix DSM of load and substation can be obtained after solving the outer layer programming. According to original matrix CM, new ownership matrix CBM can be gotten.

For a particular substation, inner optimization model is:

The decision variables are elements of matrix DBM

$$
d b m_{i j}, d b m_{i j} \in\{0,1\}
$$

Where $i=1 " n_{\text {subi }} j=1$ ' $n_{\text {load }}$

The objective function:

$$
\min \left(\sum_{k=1}^{n_{\text {subi }}} d b \max _{k}\right)
$$

Equality constraints are:

$$
\left\{\begin{array}{l}
\sum_{k=1}^{n_{\text {subi }}} d b m_{k j}=d s m_{i j}, \quad j=1 \cdots n_{\text {load }} \\
\sum_{k=1}^{n_{\text {subi }}} c b m_{k j} d b m_{i j}=d s m_{i j}, \quad j=1 \cdots n_{\text {load }}
\end{array}\right.
$$

Inequality constraints are:

$$
\left\{\begin{array}{l}
\sum_{j=1}^{n_{\text {load }}} P L_{j} d b m_{i j} \leq P_{\text {bayk }}, \quad k=1 \ldots n_{\text {subi }} \\
d b m_{k j} \leq \operatorname{dbmax}_{k}, \quad k=1 \cdots n_{\text {subi }}, j=1 \cdots n_{\text {load }}
\end{array}\right.
$$

In summary ,the essence of inner optimization model is binary integer linear programming(ILP) problem, which includes constraint of:

1) $n_{\text {subi }} X\left(n_{\text {load }}+1\right)$ integer variable as $\{0,1\}$; 
2) $n_{\text {subi }} \times\left(n_{\text {load }}+1\right)$ inequality constraints;

3) $2 \times n$ equality constraints

\section{Bay Optimization Algorithm Process Based on Hierarchical Model}

In response to these bay optimization problem, applying bay resource optimization algorithm based on hierarchical model to solve the objective function. Flow chart is illustrated by fig. 5

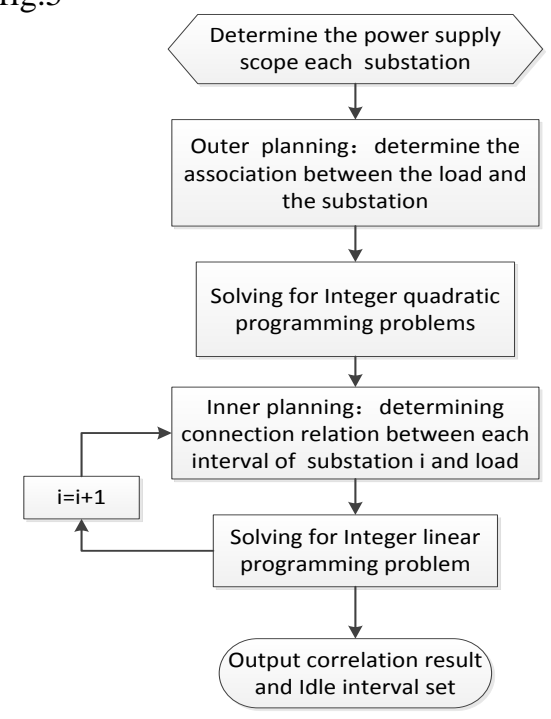

Fig.5 The flow chart of bay resource optimizationalgorithm based on hierarchical model

(1)confirming power supply scope of each substation.

(2)set up objective function and Restrictions of outer planning;

(3)solving for IQP problem of outer planning, to get correlation matrix DSM of

load and substation.

(4)Implement inner planning successively from 1 to $\mathrm{m}$ depending on matrix DSM and set up objective function and Restrictions.

(5)Solving for inner planning ILP problem to get correlation matrix DBM of load and substation bay.

(6)Output allocation results for bay resource corresponding to load in each substation.

\section{Analysis of Example}

There is a region which includes 5 substations, 25 load point and a total of 23 bay resource. Available capacity for substation I is $40 \mathrm{MW}$ and there are 4 bays, 
capacity of each bay is in the order of $[12,12,12,12](\mathrm{MW})$; Available capacity for substation II is $60 \mathrm{MW}$ and there are 6 bays, capacity of each bay in the order of $[12,12,12,12,8,8](\mathrm{MW})$; Available capacity for substation III is $30 \mathrm{MW}$ and there are 4 bays, capacity of each bay in the order of $[12,8,8,8]$ (MW); Available capacity for substation IV is 60MW and there are 6 bays, capacity of each bay in the order of $[8,8,8,12,12,12]$ (MW); Available capacity for substation $\mathrm{V}$ is $30 \mathrm{MW}$ and there are 6 bays, capacity of each bay in the order of $[8,8,8,12,12,12]$ (MW).Load needs of the this region is $131 \mathrm{MW}$ distributing in 25 load point and load of each load point is in order of $[4.8,5.2,6,5,6,8,3,2,8,6.5,9,4.5$, $10,6,5.5,8,68,4,3,2,4,1,3,2.5]$ (MW).

All elements of home range matrix $\mathrm{CM}$ of each load point are illustrated by Fig.6. Attribution matrix SM of load to substation can be obtained according to $\mathrm{CM}$ and is illustrated by Fig.7.

(1)Solving for outer planning

Associated decision matrix for load to substation is DSM and all elements are illustrated by Fig.8.

(2)Solving for inner planning

In substation, correlation decision matrix of load and bay are DBM1 DBM5, all elements are illustrated by Fig.9-Fig.13.According to matrix DBM, number of idle bay is in order of $2,1,2,2,1$ in substation I-V.

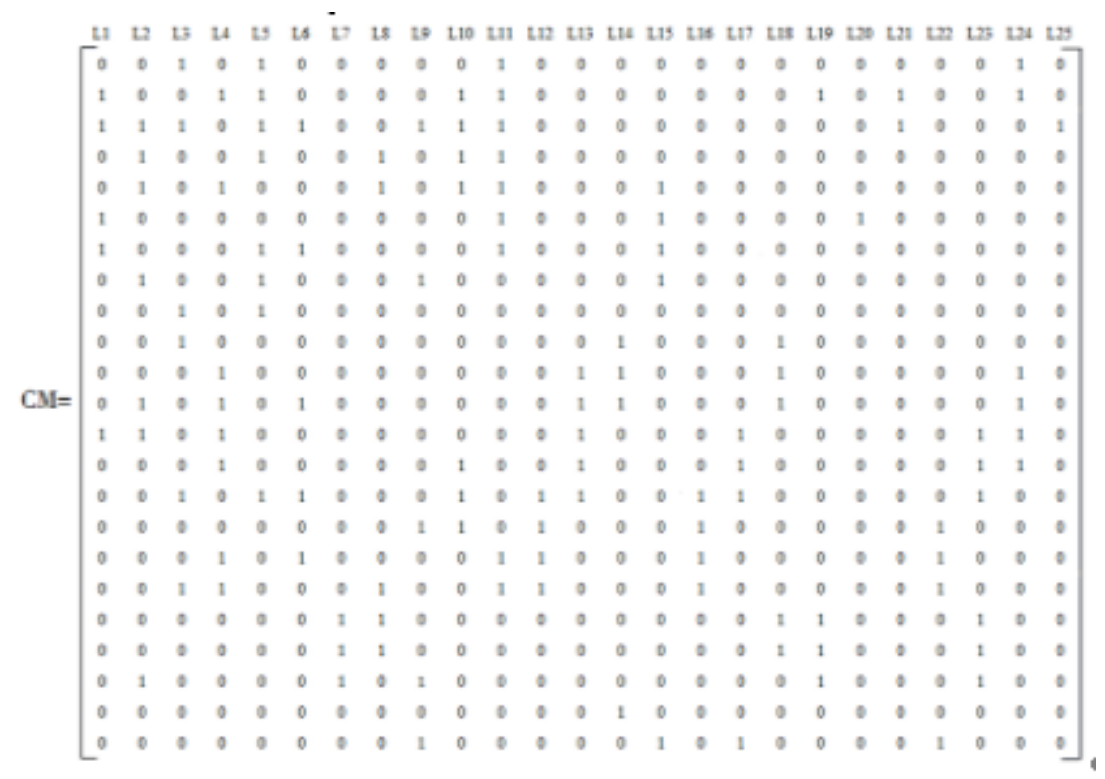

Fig.6 Elements of matrix CM 
Where line of the matrix represents number of bay.

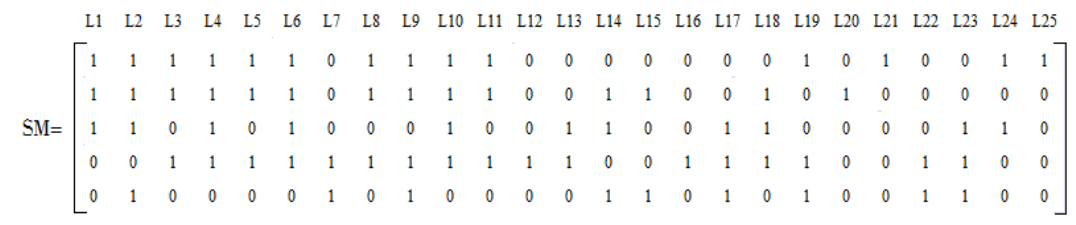

Fig.7 Elements of matrix SM

Where line of the matrix represents number of substation.

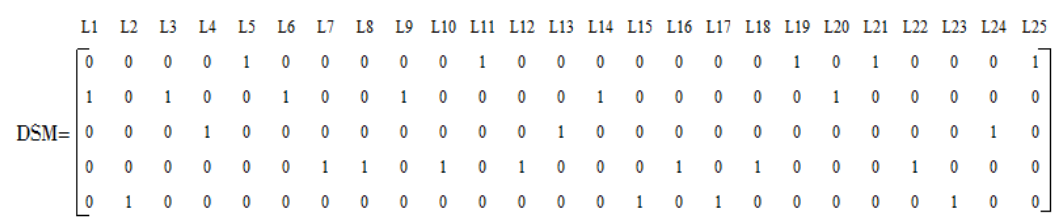

Fig.8 Elements of matrix DSM

Where line of the matrix represents number of substation.

DBM1 $=\left[\begin{array}{llllllllllllllllllllllllll}0 & 0 & 0 & 0 & 0 & 0 & 0 & 0 & 0 & 0 & 0 & 0 & 0 & 0 & 0 & 0 & 0 & 0 & 0 & 0 & 0 & 0 & 0 & 0 & 0 \\ 0 & 0 & 0 & 0 & 1 & 0 & 0 & 0 & 0 & 0 & 0 & 0 & 0 & 0 & 0 & 0 & 0 & 0 & 1 & 0 & 1 & 0 & 0 & 0 & 0 \\ 0 & 0 & 0 & 0 & 0 & 0 & 0 & 0 & 0 & 0 & 1 & 0 & 0 & 0 & 0 & 0 & 0 & 0 & 0 & 0 & 0 & 0 & 0 & 0 & 1 \\ 0 & 0 & 0 & 0 & 0 & 0 & 0 & 0 & 0 & 0 & 0 & 0 & 0 & 0 & 0 & 0 & 0 & 0 & 0 & 0 & 0 & 0 & 0 & 0 & 0\end{array}\right]$

Fig.9 Elements of matrix DBM1

Where line of the matrix represents number of bay.

DBM2 $=\left[\begin{array}{lllllllllllllllllllllllll}\text { L1 } & \text { L2 } & \text { L3 } & \text { L4 } & \text { L5 } & \text { L6 } & \text { L7 } & \text { L8 } & \text { L9 } & \text { L10 } & \text { L11 } & \text { L12 } & \text { L13 } & \text { L14 } & \text { L15 } & \text { L16 } & \text { L17 } & \text { L18 } & \text { L19 } & \text { L20 } & \text { L21 } & \text { L22 } & \text { L23 } & \text { L24 } & \text { L25 } \\ 0 & 0 & 0 & 0 & 0 & 0 & 0 & 0 & 0 & 0 & 0 & 0 & 0 & 0 & 0 & 0 & 0 & 0 & 0 & 0 & 0 & 0 & 0 & 0 & 0 \\ 1 & 0 & 0 & 0 & 0 & 0 & 0 & 0 & 0 & 0 & 0 & 0 & 0 & 0 & 0 & 0 & 0 & 0 & 0 & 1 & 0 & 0 & 0 & 0 & 0 \\ 0 & 0 & 0 & 0 & 0 & 1 & 0 & 0 & 0 & 0 & 0 & 0 & 0 & 0 & 0 & 0 & 0 & 0 & 0 & 0 & 0 & 0 & 0 & 0 & 0 \\ 0 & 0 & 0 & 0 & 0 & 0 & 0 & 0 & 1 & 0 & 0 & 0 & 0 & 0 & 0 & 0 & 0 & 0 & 0 & 0 & 0 & 0 & 0 & 0 & 0 \\ 0 & 0 & 1 & 0 & 0 & 0 & 0 & 0 & 0 & 0 & 0 & 0 & 0 & 0 & 0 & 0 & 0 & 0 & 0 & 0 & 0 & 0 & 0 & 0 & 0 \\ 0 & 0 & 0 & 0 & 0 & 0 & 0 & 0 & 0 & 0 & 0 & 0 & 0 & 1 & 0 & 0 & 0 & 0 & 0 & 0 & 0 & 0 & 0 & 0 & 0\end{array}\right]$

Fig.10 Elements of matrix DBM2

Where line of the matrix represents number of bay

DBM3 $=\left[\begin{array}{lllllllllllllllllllllllllllll}\text { L1 } & \text { L2 } & \text { L3 } & \text { L4 } & \text { L5 } & \text { L6 } & \text { L7 } & \text { L8 } & \text { L9 } & \text { L10 } & \text { L11 } & \text { L12 } & \text { L13 } & \text { L14 } & \text { L15 } & \text { L16 } & \text { L17 } & \text { L18 } & \text { L19 } & \text { L20 } & \text { L21 } & \text { L22 } & \text { L23 } & \text { L24 } & \text { L25 } \\ 0 & 0 & 0 & 0 & 0 & 0 & 0 & 0 & 0 & 0 & 0 & 0 & 1 & 0 & 0 & 0 & 0 & 0 & 0 & 0 & 0 & 0 & 0 & 0 & 0 \\ 0 & 0 & 0 & 0 & 0 & 0 & 0 & 0 & 0 & 0 & 0 & 0 & 0 & 0 & 0 & 0 & 0 & 0 & 0 & 0 & 0 & 0 & 0 & 0 & 0 \\ 0 & 0 & 0 & 0 & 0 & 0 & 0 & 0 & 0 & 0 & 0 & 0 & 0 & 0 & 0 & 0 & 0 & 0 & 0 & 0 & 0 & 0 & 0 & 0 & 0 \\ 0 & 0 & 0 & 1 & 0 & 0 & 0 & 0 & 0 & 0 & 0 & 0 & 0 & 0 & 0 & 0 & 0 & 0 & 0 & 0 & 0 & 0 & 0 & 1 & 0\end{array}\right]$

Fig.11 Elements of matrix DBM3 
Where line of the matrix represents number of bay

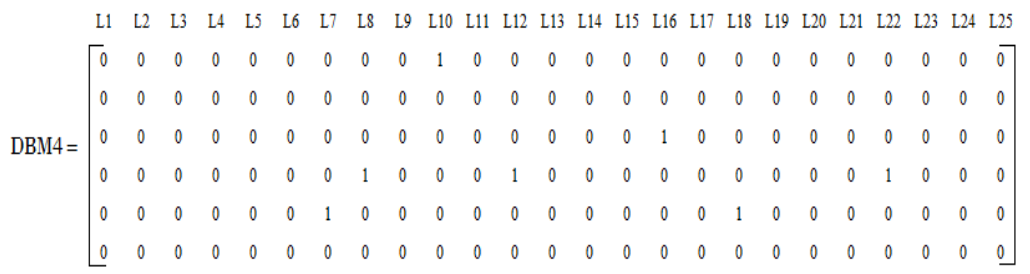

Fig.12 Elements of matrix DBM4

Where line of the matrix represents number of bay.

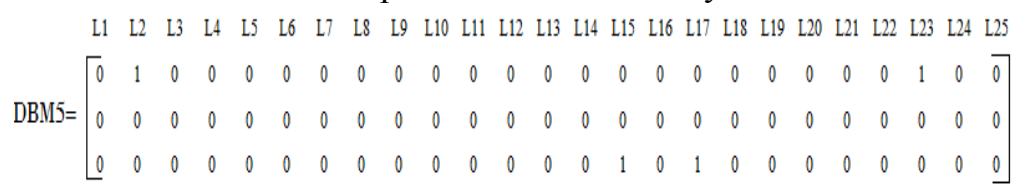

Fig.13 Elements of matrix DBM5

(3) Optimization Results

After optimization solution for example, load allocated to substation I is $23.5 \mathrm{MW}$ and load rate is $58.75 \%$ with two bays occupied and two bays remained; load allocated to substation II is $35.8 \mathrm{MW}$ and load rate is $59.67 \%$ with five bays occupied and one bays remained; load allocated to substation III is $18 \mathrm{MW}$ and load rate is $60.00 \%$ with two bays occupied and two bays remained; load allocated to substation IV is $36 \mathrm{MW}$ and load rate is $60 \%$ with four bays occupied and two bays remained; load allocated to substation $\mathrm{V}$ is $17.7 \mathrm{MW}$ and load rate is $59.00 \%$ with two bays occupied and one bays remained.

\section{Conclusion}

Contrary to shortage of substation bay resources for distribution network in at city center, inter resource optimization algorithm based on hierarchical model is proposed. Outer planning stage, the method optimizes substation load distribution as goal of balanced use of substation capacity and obtain cluster load match with each substation; inner planning stage, connection relation between load and bay is determined with goal of up to the amount of free bay and bay resource optimization configuration program with maximum utilization for substation bay resources can be gotten. Apply the proposed optimization algorithm for a practical example to optimize substation bay resource configuration. The simulation results show the method can use existing bay resource reasonably and fully while combining substation bay power supply capacity. It has important signifi- 
cance for network planning and construction work to improve the reliability of power supply and distribution network transmission capacity

\section{References}

1. WANG Guocheng, WU Risheng, HE Yisi, et al. Discussion on urban distribution layout and construction[J]. Power System Protection and Control, 2005, 33(20): 74-78

2. HUO Kailong, WANG Zhuding, CHANG Gang, et al. A practical method for medium voltage distribution network planning in target year[J]. Power System Technology, 2013, 37(6): 1769-1774

3. SONG Yunting, ZHANG Dongxia, WU Junling, et al. Comparison and Analysis on Power Supply Reliability of Urban Power Distribution Network at Home and Abroad[J]. Power System Technology, 2008, 32(23): 13-18 (in Chinese) .

4. CAI Zexiang, ZHOU Quan, et al. Influence of Distribution System Connection Modes on Power Supply Reliability[J]. Proceedings of the CSU-EPSA, 2008, 32(23):13-18 (in Chinese) .

5. WU Xiong, WANG Xiuli, et al. Economic Generation Scheduling of a Microgrid using Mixed integer programming[J]. Proceedings of the CSEE, 2013, 33(28):1-8 (in Chinese) .

6. XU Dan, XIA Shaolian, et al. Fast unit commitment based on heuristic mixed integer programming [J]. Proceedings of the CSEE, 2012. 40(21): 17 (in Chinese) .

7. ChENG Lin, HE Jian, SUN Yuanzhang. Impact of transmission line'srealtime reliability model parameter upon power system operationalreliability evaluation. Power System Technology, vol 13, pp.8-13.Jan 2006

8. SUN Yanyan“. Shandong 500kv power grid reliability evaluation" PostGraduate dissertation. Univ SDU.2007.

9. SONG Xioatong, TAN Zhengyu. Application of Improved Importance Sampling Method Power System Reliability Evaluation. Power System Technology.vol 29. pp.56-59. Jul. 2005

10. ZHOU Jianqi, ZHAO Xia. Modelsand methodsapplied torisk assessment ofpower systems. ELECTRIC POWER.vol 39.pp.77-81Aug 2006. 